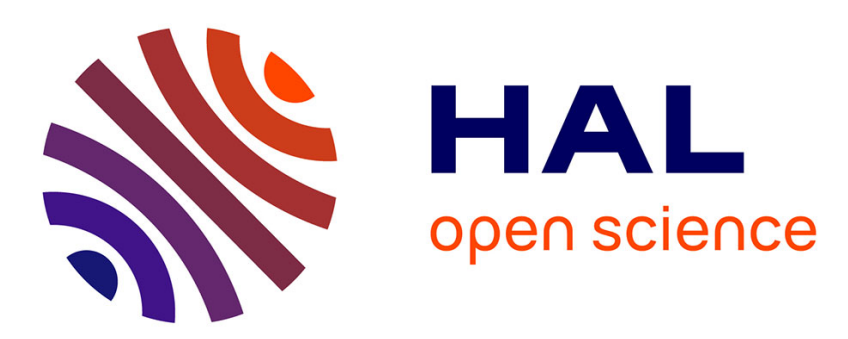

\title{
Finite-element modeling of the electro-thermal contacts in the spark plasma sintering process
}

Charles Manière, Anthony Pavia, Lise Durand, Geoffroy Chevallier, Khalid Afanga, Claude Estournès

\section{- To cite this version:}

Charles Manière, Anthony Pavia, Lise Durand, Geoffroy Chevallier, Khalid Afanga, et al.. Finiteelement modeling of the electro-thermal contacts in the spark plasma sintering process. Journal of the European Ceramic Society, 2016, 36 (3), pp.741-748. 10.1016/j.jeurceramsoc.2015.10.033 . hal01727546

\section{HAL Id: hal-01727546 \\ https://hal.science/hal-01727546}

Submitted on 28 Nov 2020

HAL is a multi-disciplinary open access archive for the deposit and dissemination of scientific research documents, whether they are published or not. The documents may come from teaching and research institutions in France or abroad, or from public or private research centers.
L'archive ouverte pluridisciplinaire HAL, est destinée au dépôt et à la diffusion de documents scientifiques de niveau recherche, publiés ou non, émanant des établissements d'enseignement et de recherche français ou étrangers, des laboratoires publics ou privés. 


\title{
Finite-element modeling of the electro-thermal contacts
}

\section{in the Spark Plasma Sintering process}

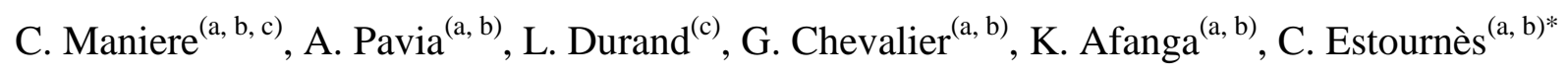

(a) Université de Toulouse, Institut Carnot CIRIMAT, UPS CNRS, Université PaulSabatier, 118 route de Narbonne 31062 Toulouse cedex 9, France

(b) CNRS, Institut Carnot CIRIMAT, 118 route de Narbonne 31602 Toulouse Cedex 9, France

(c) Cemes, CNRS UPR 8011 et Université de Toulouse, 29 rue Jeanne Marvig, 31055 Toulouse, France

\section{Keywords}

Spark-Plasma-Sintering; Electrical and Thermal Contact; Papyex; Finite Element Method

\begin{abstract}
Spark plasma sintering (SPS) is a breakthrough process for powder consolidation assisted by pulsed current and uniaxial pressure. In order to model the temperature variations of the tools during a SPS cycle, the Graphite-Papyex-Graphite contact phenomena are studied experimentally and modeled by finite element calculations. Compared to conducting materials, the thermo graphic image of an insulating sample (alumina) shows strongly localized heating along the Papyex implying contact effects are predominant. The aim of this modeling study is to determine the main contact phenomena due to Papyex. It is based on numerous experimental data and studies the case of alumina sintering. Finally the contact model is confronted to experimental thermal images.
\end{abstract}

\footnotetext{
* Corresponding authors:
}

Claude ESTOUNES: CIRIMAT 118 route de Narbonne 31062 Toulouse, France

Tel: (+33) 561556109; Fax: (+33) 561556163

E-mail address: estournes@ chimie.ups-tlse.fr 


\section{Introduction}

Spark Plasma Sintering (SPS) is essentially a powder consolidation process assisted by pulsed current and uniaxial pressure. It allows sintering of refractory materials in few minutes instead of days by free sintering [1]. The main goal of the thermal modeling of the process is to determine the temperature distribution in the tools and the sample, to experimentally explain any microstructural variations observed, and in the long term to minimize them [2-3-4]. The SPS column (tools + spacers) detailed in Figure 1 is usually composed of graphite, to ensure good electrical contact and suitable friction between the inner sliding parts of the tools, a flexible graphite sheet (Papyex ${ }^{\circledR}$ from Mersen) is introduced at the top and bottom of the SPS column and around the sample. The number of papers published on the simulation of the SPS process has drastically increased since the 2000's as has the development of Finite Element Modeling (FEM) software. The first simulations were only devoted to the electro-thermal behavior of the tool (i.e. not the entire column), with or without the presence of the Papyex but not considering its impact on the temperature distribution [5-6-7]. These simulations allow us to understand the general distribution of the current and the temperature gradient. With the work of Matsugi et al in 2003 [8-9] SPS modeling started to show a better correlation between the calculated and experimental temperatures

From 2003 until today SPS modeling has made a lot of progress. The models now include more parts of the SPS column and more physics as for example in the work of Olevsky et al where the chamber of the SPS and the densification of the sample are modeled simultaneously [10-11]. But in most of these works, the contact resistances generated by the presence of the carbon sheet are not considered.

However, few authors have already made in-situ measurements of the electrical contacts resistance (ECR) in the SPS [12-13], others authors determine the ECR by calibration [1415]. But using their values, in our model, it is difficult to obtain good experimental accordance because the properties of the contact change with pressure and temperature [16]. 
In this study we used inverse analysis to identify the contact phenomena at all interfaces of the tool using the temperature distribution revealed experimentally by thermal imaging [17] and/or using thermocouples located at different points of the tool. This work is based on several modeling studies performed at the CIRIMAT and CEMES laboratories on the same configuration of the SPS column [2-3-4].

\section{Materials and methods}

A first set of SPS experiments has been performed using an open die (i.e.: a slice was removed) to reveal the internal temperature distribution and to highlight the predominant effects of the electric and thermal contacts for both insulating and conductive samples. Others experiments were made using full die to perform temperature measurements at several points to understand and calibrate the main contact resistances responsible of the high thermal effects revealed by the open die experiments.

\subsection{Thermal images on open dies}

All the SPS experiments were made on a Dr. Sinter 2080, SPS Syntex Inc, Japan, SPS machine at the "Plateforme Nationale CNRS de Frittage Flash" located at University Toulouse III-Paul Sabatier. The thermal images reported in Figure 1 were acquired with an infrared camera (FLIR SYSTEMS SC6000) [17]. In this configuration, open molds were used to experimentally measure the internal temperature distribution around the sample. Graphite foils (Papyex) were placed at both interfaces punch/sample and to cover the inner wall of the die (Figure 2). Figure 1 shows at low temperature two different cases where pellets of insulating and conducting materials, respectively alumina and copper, were loaded into the die. All the experiments were made on fully dense samples to avoid having to model sintering in the following part.

\subsection{Temperature measurements for ECR and TCR calibrations}


A double Papyex is classically introduced at both extremities of the SPS column (interfaces spacer/electrode figure 2) to ensure a good electrical contact between the inconel electrodes and the graphite spacers. A first experiment was performed with only a graphite part $(20 \pm 0.05$ $\mathrm{mm}$ in diameter and $20.55 \pm 0.05 \mathrm{~mm}$ height) placed between the spacers to calibrate first this spacer/electrode contact resistance. A control thermocouple was located in the graphite part at a depth of 3mm (Figure 2a). A second thermocouple was placed on the upper spacer to calibrate the external thermal contact due the double Papyex foils present between the spacer and the inconel (Figure 2a). High applied pressure (100MPa) was used to avoid any additional contact phenomena between the eentrat graphite part and the spacers.

To calibrate the electrical and thermal contacts around the sample, an experiment similar to that used to obtain the thermal images was performed in a closed mold (Figure 2b). Two thermocouples were introduced (Figure 3), one in the die at a depth of $3 \mathrm{~mm}$ from its external surface to monitor the SPS temperature and the second inside the die in contact with the graphite foil to calibrate the contact phenomena linked to the use the Papyex sheet. To measure RMS values of pulsed currents a Rogowski coil sensor is used [17].

The calibration of the different ECR and TCR at the interfaces underlined in the two configurations reported in figure 2, were performed step by step using an electro-thermal model developed on a finite element code (COMSOL) that will be described in the following section.

\section{Theory / Calculation: Electro-thermal model.}

The Joule heating model is built up with two main concepts, the current distribution is determined by partial differential equation (1).

$$
\nabla \cdot \vec{J}=\nabla \cdot(\sigma \vec{E})=\nabla \cdot(-\sigma \nabla U)=0
$$

secondly the temperature distribution is determined by the heat equation (2). 


$$
\nabla \cdot(-\lambda \nabla T)+\rho C p \frac{\partial T}{\partial t}=J E
$$

Where $J$ is the current density, $E$ the electric field, $U$ the electric potential and for each materials of the device (Figure 2), $\sigma$ the electric conductivity, $\lambda$ the thermal conductivity, $\rho$ the density, $C p$ the calorific capacity and $T$ the absolute temperature.

There are two main thermal limit conditions:

(i) A radiative flux on the vertical wall of spacers, die, punches and electrodes governed by Eq. 3 .

$$
\phi_{r}=\sigma_{s} \cdot \varepsilon \cdot\left(T_{e}^{4}-T_{a}^{4}\right)
$$

Where $\sigma_{s}$ is the Stefan-Boltzmann's constant, $\phi_{r}$ the radiative heat flux, $T_{e}$ the emission surface temperature, $T_{a}$ the chamber wall temperature, $\varepsilon$ the emissivity ( 0.8 for the graphite and 0.67 for the inconel [3]).

$$
\phi_{r}=\sigma_{s} \cdot \varepsilon \cdot\left(T_{e}^{4}-T_{a}^{4}\right)
$$

(ii) A conducto-convective flux on the horizontal wall of the inconel near the water cooling system (see Figure 2) is considered and governed by Eq. 5 .

$$
\phi_{c}=h_{c} \cdot\left(T_{i}-T_{w}\right)
$$

Where $\phi_{c}$ the conducto-convective heat flux, $T_{i}$ the wall surface inconel temperature, $T_{w}$ the water temperature, $h_{c}$ the conducto-convective coefficient $\left(880 \mathrm{~W} \cdot \mathrm{m}^{-2} \cdot \mathrm{K}^{-1}\right.$ at the level of the inconel [2]).

The properties of the materials considered are given in tables I and II.

The Electric Contact Resistance (ECR) and Thermal Contact Resistance (TCR) were, as a first approximation, introduced in the model as constant values in order to roughly understand 
their effects on the phenomena of temperature gradients experimentally observed. The TCR was introduced in the model using an interfacial condition that simulates the effect of an equivalent thin layer of thickness $e=0.1 \mathrm{~mm}$ of thermal conductivity $\mathrm{h}_{\mathrm{c}}$ in $\mathrm{W} / \mathrm{mK}$, which obeys the relation:

$$
-\boldsymbol{n}_{\boldsymbol{u}} \cdot\left(-K_{d} \nabla T_{d}\right)=h_{c} \frac{\left(T_{u}-T_{d}\right)}{e}
$$

Where $K_{d}$ is the thermal conductivity of the material considered, the source and destination contact surfaces are indicated by the suffixes $u$ and $d, \boldsymbol{n}$ is the normal surface.

The ECR graphite/Papyex/graphite is mainly due to the introduction of this graphite layer of thickness $0.2 \mathrm{~mm}$ which has anisotropic electric (1E-5 $\Omega . \mathrm{m}$ in plane and $5 \mathrm{E}-4 \Omega . \mathrm{m}$ through the thickness) and thermal properties $(150 \mathrm{~W} /(\mathrm{m} . \mathrm{K})$ in plane end $5 \mathrm{~W} /(\mathrm{m} . \mathrm{K})$ through the thickness) [18-19]. To introduce this ECR in the model, the basic properties of this graphite layer are subsequently modified step by step with successive calibrations. The ECR is thus simulated by adding a constant value $\left(\rho_{0}\right)$ to the electric resistivity of graphite in the radial direction and the constant part of the in-plane thermal conductivity of the layer is multiplied by a factor mpl to model the thermal anisotropy.

A scheme of the full die used for the calibration experiment is given in figure 3 where all the locations of the boundary conditions used for the FEM simulation are reported.

\section{Results and discussion}

\subsection{ECR and TCR at the Inconel/Spacer interface}

The Inconel/Spacer Interface is the place of a huge exchange of heat (evacuation of calories) from the SPS column to the cooling system located on each of the electrodes. To ensure a good electrical contact between the Inconel parts and the spacers a double sheet of graphite foils (Papyex) was placed between them. Note that even though the applied pressure on the die is of the order of hundred MPa, the pressure at this interface remains small (less than $10 \mathrm{MPa}$ in our configuration). This Inconel/2*graphite foils/Spacer interface is where the 
thermal contact resistance (TCR) is determined, and may strongly influence heat transfer at this level. A set of experiments were then conducted using the configuration reported in Fig 2a to calibrate the contact of this interface before starting the inverse analysis of the contacts of the other interfaces present in the mold.

The variation of the temperatures measured on the spacer and the heated graphite part are reported in Figure 4 alongside the simulated ones. The value of the latter was calibrated by adjusting the TCR (TCR/PapyexX2/TCR assembly) until perfect concordance of the temperature was attained (Figure 4a).

The results show that the correct value of the TCR is $0.04 \mathrm{~W} / \mathrm{m} . \mathrm{K}$, the thermal conductivity of an $0.1 \mathrm{~mm}$ equivalent thin layer.

The simulated temperature of the column (Figure $4 \mathrm{~b}$ ) shows that the graphite part is, as expected, the place of the highest temperature and that the difference of color at the Spacer/Inconel interface is characteristic of the TCR created by the double Papyex layer.

The electrical contact resistance (ECR) of this interface also exists but because it is an area of large diameter, the current density and consequently the heat dissipated is very low. For this reason we have chosen to ignore the ECR at this place, since its thermal contribution appears to be very small.

\subsection{ECR and TCR at the die interfaces.}

4.2.1. Contacts due to the graphite foil around the sample.

An experimental design has already been performed by Maniere et al. which revealed that the ECR decreases with temperature and applied pressure to values close to zero above $800^{\circ} \mathrm{C}$ and $50 \mathrm{MPa}[18]$.

These values are mostly exceeded in SPS cycles for densification of ceramics, where pressure and temperature are usually of the order of $100 \mathrm{MPa}$ and $1000{ }^{\circ} \mathrm{C}$. Thus according to 
Anselmi-Tamburini et al [16], it is justified to ignore the effect of any ECR in the area surrounding the sample which is subjected to high pressures and temperatures.

\subsubsection{Contacts through the graphite foil located on the inner wall of the die.}

Thermal images taken on open dies show that for an insulating sample (alumina), Papyex undergoes strong localized heating (Figure 1a) while for a conducting material (copper) this is not the case (Figure 1b). Moreover, it is interesting to note that unexpectedly [6-9] in increasing temperature regime the punches are cooler than the die and the maximum temperature is observed at the level the sample where a hot spot is revealed (for alumina sample). In contrast, in a conducting sample (copper) the temperature distribution shows a lower effect of the contact due to the Papyex probably because the current mainly passes through the punch and sample. The punches are in this case the place of maximum temperature (Figure 1b).

To quantify the heat generated by the current going through the Papyex and the influence of the different contact phenomena, we conducted the experiment in a closed die according the configuration reported in Figure 2b. The time dependence of the two temperatures measured is reported in Figure 5, where the blue represents the set temperature given by the control thermocouple, the dwell temperature was fixed at $1000{ }^{\circ} \mathrm{C}$. The green curve represents the temperature measured by the thermocouple in contact with the Papyex and it appears to be significantly higher than the set point and attains approximately $1120{ }^{\circ} \mathrm{C}$ at the beginning of the dwell. The Papyex temperature is about $120{ }^{\circ} \mathrm{C}$ higher than that measured by the control thermocouple. This confirms the thermal image obtained with the open die containing the alumina sample Figure 1a and the localized heating on the Papyex.

\subsubsection{Modeling without contact}


A first $2 \mathrm{D}$ axisymmetric model considering the measured $\mathrm{I}_{\mathrm{rms}}$ as the input data and the bulk materials data from the suppliers and given in Table I and Table II, shows (Figure 6a) a general temperature distribution that is more homogeneous than that observed experimentally using the infrared camera. As shown in Figure 6a the simulated temperature corresponding to the two experimental curves shows differences of only $10{ }^{\circ} \mathrm{C}$. Moreover, the dwell temperatures simulated for the control and the Papyex thermocouples are respectively 100 and $200^{\circ} \mathrm{C}$ lower than those measured experimentally.

These differences could be related to the fact that in this first simulation neither the ECR nor the TCR of the vertical contact of the Graphite/Papyex/Graphite interface were considered. As a consequence, the temperatures simulated are underestimated because the heat dissipated by the ECR is not taken into account.

In the following sections, an inverse analysis is performed to calibrate the ECR and TCR of the vertical contact Graphite/Papyex/Graphite based on the experimental temperatures given at the thermocouples (Figure 5). The principle is that the information sought on the phenomena of contact can be obtained by re-calculating the differences in temperatures observed and modeled.

4.2.4. Modeling considering the electrical contact through the graphite foil

To correct the temperature in the region of the control thermocouple (blue curve Figure 6b) an equivalent $\mathrm{ECR}$ value of $\rho_{0}=2.83 \times 10^{-4} \Omega \mathrm{m}$ was added to the radial electric resistivity of the 0.2 mm layer introduced at the Graphite/Papyex/Graphite contact. However, the model is not satisfactory as the temperature distribution in the mold remains too homogeneous compared to that observed on the open die experiment (Figure 1a). The differences between the simulated temperature curves at dwell were only about $40^{\circ} \mathrm{C}$ (Figure $6 \mathrm{~b}$ ), i.e. far below the $120^{\circ} \mathrm{C}$ observed experimentally. Furthermore, the Papyex does not seem to undergo localized 
heating (Figure 6b). By varying the amplitude of the electrical resistance in the thickness or even in the plane of Papyex, localized heating of the Papyex is still unattained. So, in addition to the ECR, a TCR on both sides of the Papyex should be taken into account to explain such differences in temperature.

4.2.5. Modeling the electrical and thermal contacts engendered by the graphite foil.

The TCR is simulated on both sides of the papyex by a boundary condition that is defined as an equivalent thin layer of $0.1 \mathrm{~mm}$ and of thermal conductivity $\mathrm{h}_{\mathrm{c}}$ (red lines figure 3 ). Unlike the ECR, the TCR is modeled in this way in order to maintain the heat generated by the ECR inside the layer. We consider the vertical papyex thermal contact perfect in the area in contact with the sample. This hypothesis is take because of the high pressure applied on the sample responsible of a high radial pressure on the contact sample/die. The calibration of the temperature at the dwell of the Papyex (green curve Figure 6c) gives a value of $h_{c}=0.01$ $\mathrm{W} /(\mathrm{m} . \mathrm{K})$. As Figure 6c shows, considering the TCR the heat seems to be more localized in the Papyex and the punches are cooler than the die. The TCR seems paramount, against all expectations, to maintain heat in the Papyex.

Two points of disagreement remain between the simulation and the experimental observations:

- The simulation shows a high thermal gradient along the Papyex that is not present in the experiment (see Figure 1a).

- The hot spot present around the sample figure 1a does not appear in the modeled die.

When the simulation is performed without considering the electric contact, the distribution of the electric power density dissipated is maximum in the punches (Figure 7a) and not along the Papyex. In contrast, with ECR the modeled current lines (Figure 7b) show, inter alia, a high concentration of current at the upper extremity of the graphite foil. At the same time, the 
modeled electric power density dissipated shows its maximum at the same level in good agreement with the modeled thermal gradient (Figure 7c). Consequently, the thermal gradient along the Papyex observed in the model is essentially due to the electrical part of the model.

Another parameter needs to be varied to correct this gradient and to model the hot spot. Varying the electrical and thermal contact and properties of Papyex in the plane or in the thickness failed to model the hot spot. Only a significant increase $(\mathrm{mpl}=10)$ of the constant part of the in-plane thermal conductivity of the Papyex can both make the temperature distribution along the Papyex more homogeneous and generate the hot spot in the sample (Figure 8) while maintaining a good agreement between the modeled and the measured temperatures. The manufacturer claims that the Papyex in-plane thermal conductivity can be greatly increased with its density [19]. Before each SPS run, the graphite foils is rolled prior to its introduction inside the mold to adapt its thickness to the gap between the punches and the die and to have a better sliding contact. So, it is reasonable to consider such an increase of its thermal conductivity in the simulations.

The final data for the contact phenomena of the vertical contact are: $\rho_{0}=2.23 \times 10^{-4} \Omega . \mathrm{m}$, $\mathrm{h}_{\mathrm{c}}=0.01 \mathrm{~W} /(\mathrm{m} . \mathrm{K})$ and the constant part of the Papyex in-plane thermal conductivity is increased by an order of magnitude (x10).

To validate the data determined in the previous sections and to test the robustness of the model, a 3D simulation corresponding to the experiment on the open die containing a dense alumina pellet (reported in fig 1a) was performed. In this simulation the cut faces have an emissivity of 0.8 for the graphite parts and also 0.8 for the alumina [17]. A $100 \mathrm{~K} / \mathrm{min}$ heating rate and a applied pressure of $100 \mathrm{MPa}$ are used. It is to be noted that a high pressure is used without failure of the cut die, this result is possible because the sample is densify before the experiment. The results of this simulation are given in figure 9. The following observations can be made: 
i) First, in this model a high concentration of heat is preserved along the Papyex as in the experiment.

ii) The hot spot is modeled in the vicinity of the sample.

iii)The general modeled distribution of temperature shows that the punches are cooler than the die, an experimental fact not previously predicted by our conventional models without contact.

As a result, all the singularities observed on the thermogram of the open die containing the alumina pellet are generated by the 3D simulation and the magnitudes of the simulated gradients are in agreement with the experimentally observed ones.

\section{Conclusion}

Electro-thermal simulation of the SPS process was conducted by finite element modeling. The use of materials properties provided by the manufacturers did not allow us to faithfully reproduce the temperatures and thermal gradients found experimentally in open matrices. Considering the electrical and thermal contacts at the different interfaces present in the SPS stack, simulation gave more realistic modeling of the temperature and gradients at all points of the die. To summarize, the main effects are listed below:

- The electrical contact resistances are responsible for a temperature raise of the overall system (die, punches and sample) of around $100{ }^{\circ} \mathrm{C}$.

- The thermal contact resistance on both sides of the Papyex maintain a small part of the heat generated inside it thus explaining the localized heating observed in the thermal image along the Punch/Die interface.

- The in-plane thermal conductivity of the Papyex appears to be dramatically increased probably due to the lamination step performed before its introduction in the die. This, 
is surely responsible for the hot spot present in the die near the sample observed in the thermal image.

Modifying these three key parameters allowed us to simulate the complex temperature distribution experimentally observed in the parts around the sample. It is to be noted that this methodology is very simple since it uses only few temperature data, collected in some strategic places, for calibrating the contact phenomena that are very difficult to obtain by exsitu measurements at high temperatures.

The next step, that will be the object of future work, will be to introduce the thermal contact resistance Sample/Graphite to model the temperature of the overall system in greater depth. 


\section{Acknowledgements}

The support of the LAPLACE for the instrumentation and fruitful discussions and the

Plateforme Nationale CNRS de Frittage Flash (PNF2/CNRS) are gratefully appreciated. C.M and C.E. thank the French National Research Agency (ANR) for the financial support of this study within the project ANR09 MAPR-007 Impulsé 


\section{References:}

[1] Munir Z.A, Anselmi-Tamburini U, Ohyanagi M. The effect of electric field and pressure on the synthesis and consolidation of materials: A review of the spark plasma sintering method. J Mater Sci. 41 (2006) 763-777.

[2] Molénat G, Durand L, Galy J, Couret A. Temperature control in Spark Plasma sintering : an FEM approach. Journal of Metallurgy, Volume 2010 Article ID 145431 (2010)

[3] Voisin Th, Durand L, Karnatak N, Le Gallet S, Thomas M, Le Berre Y, Castagné J.F, Couret A. Temperature control during Spark Plasma Sintering and application to up-scaling and complex shaping. J. Mater. Process. Technol. 213 (2013) 269-278.

[4] Pavia A, Durand L, Ajustron F, Bley V, Chevallier G, Peigney A, Estournes C. Electrothermal measurements and finite element method simulations of a spark plasma sintering device. J. Mater. Process. Technol. 213 (2013) 1327-1336.

[5] Raichenko A.I, Chernikova E.S. A mathematical model of electric heating of the pourous medium using current-supplying electrode/punches. Powder Metall. Met. Ceram.. 38 (1989).265-371.

[6] Yoneya M, Ikeshoji T. A numerical calculation method advantageous for complex boundary problems: An application to the pulse discharge sintering process. Mater. Trans. 11 (2001) 2165-2171.

[7] Yucheng W, Zhengyi F. Study of temperature field in spark plasma sintering. Mater. Sci. Eng., B 90 (2002) 34-37.

[8] Matsugi K, Kuramoto H, Hatayama T, Yanagisawa O. Temperature distribution at steady state under constant current discharge in spark sintering process of $\mathrm{Ti}$ and $\mathrm{Al}_{2} \mathrm{O}_{3}$ powders. $\mathrm{J}$. Mater. Process. Technol. 134 (2003) 225-232. 
[9] Matsugi K, Kuramoto H, Yanagisawa O, Kiritani M. A case study for production of perfectly sintered complex contacts in rapid consolidation by spark sintering. Mater. Sci. Eng., A 354 (2003) 234-242.

[10] Olevsky E.A. Theory of sintering: from discrete to continuum. Mater. Sci. Eng., R 23 (1998) 41-100.

[11] Olevsky E.A, Garcia-Cardona C, Bradbury W.L, Haines C.D, Martin D.G, Kapoor D. Fundamental aspects of spark plasma sintering II: Finite element analysis of scalability. J. Am. Ceram. Soc. 95 (2012) 2414-2422.

[12] Zavaliangaos A, Zhang J, Krammer M, Groza J.R. Temperature evolution during field activated sintering. Mater. Sci. Eng., A. 379 (2004) 218-228.

[13] Zhang J. Phd Thesis, Numerical simulation of thermoelectric phenomena in field activated sintering. Drexel university, Philadelphia (2004).

[14] Vanmeensel K, Laptev A, Vleugels J, Van Der Biest O. Modelling of the temperature distribution during field assisted sintering. Acta Mater. 53 (2005) 4379-4388.

[15] McWilliams B.A. Phd Thesis, Numerical simulation of electric field assisted sintering. Drexel university, Philadelphia. (2008).

[16] Anselmi-Tamburini U, Gennari S, Garay J.E, Munir Z.A. Fundamental investigations on the spark plasma sintering/synthsis process II. Modeling of current and temperature distributions. Mater. Sci. Eng. A 394 (2005) 139-148.

[17] Pavia A. Phd Thesis, Etude fondamentales pour la compréhension des mecanismes des matériaux par la technologie Spark Plasma Sintering. Paul Sabatier Univesity, Toulouse (2012).

[18] Maniere C, Pavia A, Durand L, Chevallier G, Bley V, Afanga K, Peigney A, Estournes C. Pulse analysis and electric contact measurements in Spark Plasma Sintering. Electr Pow Syst Res 127 (2015) 307-313. 
[19] https://www.mersen.com/uploads/tx_mersen/12-PAPYEX-graphite-expanse-Mersen.pdf (2015).

[20] Conrad H, Sprecher AF, Cao W.D , Lu X.P. Electroplasticity the effect of electricity on the mechanical properties of metals. J. Mater. Sci. 42 (1990) 28-33.

[21] https://www.mersen.com/uploads/tx_mersen/15-speciality-graphite-materials-forsintering-Mersen_10.pdf (2015). 
APPENDICES

Table I: Properties of Inconel and graphite (with $\mathbf{T}$ in Kelvin)

\begin{tabular}{|c|c|c|c|}
\hline & Unit & Inconel & Graphite [21] \\
\hline Heat capacity & $\mathrm{C}_{\mathrm{p}}\left(\mathrm{J} \cdot \mathrm{kg}^{-1} \cdot \mathrm{K}^{-1}\right)$ & $\begin{array}{c}344+2.50 \times 10^{-1} \mathrm{~T} \\
{[20]}\end{array}$ & $34.27+2.72 \mathrm{~T}-9.60 \times 10^{-4} \mathrm{~T}^{2}$ \\
\hline $\begin{array}{l}\text { Thermal } \\
\text { conductivity }\end{array}$ & $\lambda\left(\mathrm{W} \cdot \mathrm{m}^{-1} \cdot \mathrm{K}^{-1}\right)$ & $10.1+1.57 \times 10^{-2} \mathrm{~T}$ & $\begin{array}{c}123-6.99 \times 10^{-2} \mathrm{~T}+1.55 \times 10^{-5} \\
\mathrm{~T}^{2}\end{array}$ \\
\hline Electrical resistivity & $\rho_{\mathrm{e}}(\Omega . \mathrm{m})$ & $\begin{array}{c}9.82 \times 10^{-7}+1.6 \times 10^{-} \\
\frac{\mathrm{T}}{[4]}\end{array}$ & $\begin{array}{c}1.70 \times 10^{-5}-1.87 \times 10^{-8} \mathrm{~T} \\
+1.26 \times 10^{-11} \mathrm{~T}^{2}-2.44 \times 10^{-15} \mathrm{~T}^{3}\end{array}$ \\
\hline Density & $\rho\left(\mathrm{kg} \cdot \mathrm{m}^{-3}\right)$ & $\begin{array}{c}8430 \\
{[4]}\end{array}$ & $1904-0.01414 \mathrm{~T}$ \\
\hline
\end{tabular}

Table II: Properties of samples: alumina (with T in Kelvin)

\begin{tabular}{ccc}
\hline & Unit & Alumina [4] \\
\hline Heat capacity & $\mathrm{C}_{\mathrm{p}}\left(\mathrm{J}^{1} \mathrm{~kg}^{-1} \cdot \mathrm{K}^{-}\right.$ & 850 \\
Thermal conductivity & $\lambda\left(\mathrm{W} \cdot \mathrm{m}^{-1} \cdot \mathrm{K}^{-1}\right)$ & $39500 \mathrm{~T}^{-1.26}$ \\
Electrical resistivity & $\rho_{\mathrm{e}}(\Omega \cdot \mathrm{m})$ & $8.70 \times 10^{19} \mathrm{~T}^{-4.82}$ \\
Density & $\rho\left(\mathrm{kg} \cdot \mathrm{m}^{-3}\right)$ & 3899 \\
\hline
\end{tabular}




\section{Figure caption:}

Figure 1: Infrared Thermal Images of open die containing: a) Alumina sample b) Copper sample.

Figure 2: Schemes of the SPS configurations used : a) for Inconel/Spacer RC calibration; b) for Punch/Die RC calibration.

Figure 3: Scheme of the SPS column where the boundaries conditions and thermocouples positions used in the FEM simulations are reported.

Figure 4: Inconel/Graphite contact calibration experiment: a) evolution of the experimental and simulated temperatures of the punch and spacer during the thermal cycle b) vertical section temperature map $\left({ }^{\circ} \mathrm{C}\right)$ at the dwell given by the simulation.

Figure 5: Evolution of the experimental temperatures given by the two thermocouples, located in a full die according to the scheme given figure 2, at the contact of the graphite foil covering the inner wall and at the surface of the die.

Figure 6: Evolution of the experimental and simulated (with a 2D axisymmetric model) of both temperatures (papyex and die) during the thermal cycle (top) and vertical section temperature map at the dwell given by the simulation (bottom) : a) without contact b) with electric contact c) with electric and thermal contact.

Figure 5: 2D axisymmetric model without contact: a) Temperature distribution at dwellb) Experimental/Simulated curves of the thermocouples. 
Figure 6: 2D axisymmetric model with electric contact: a) Experimental/Simulated curves of the thermocouples b) Temperature distribution at dwell.

Figure 7: 2D axisymmetric model with electric and thermal contact a)

Experimental/Simulated curves of the thermocouples b) Temperature distribution at dwell.

Figure 7: Dwell 2D axis-symmetric modeling at the vicinity of the punch/spacer interface : a) electric current power density dissipated without electric and thermal contacts ; b)

temperature map and current lines with calibrated electric and thermal contacts ; c) electric power density dissipated with calibrated electric and thermal contacts. a) Electric power density dissipated at the vicinity the punch-spacer interface given by $2 \mathrm{D}$ modeling without taking any electric or thermal contact (a); Temperature Map and current lines simulated with electric and thermal contact (b) and Electric power density dissipated at the vicinity the punch-spacer interface by $2 \mathrm{D}$ modeling considering electric and thermal contact.

Figure 8: Vertical section temperature map at the dwell given by a 2D axisymmetric model considering electric and thermal contact and the corrected thermal conduction of the papyex (a:top) and evolution of the experimental and simulated of both temperatures (papyex and die) during the thermal cycle (b:bottom).

Figure 9: Temperature map, of the open die containing the dense alumina sample, given by the final 3D modeling. 
Fig. 1 (single-column fitting image)
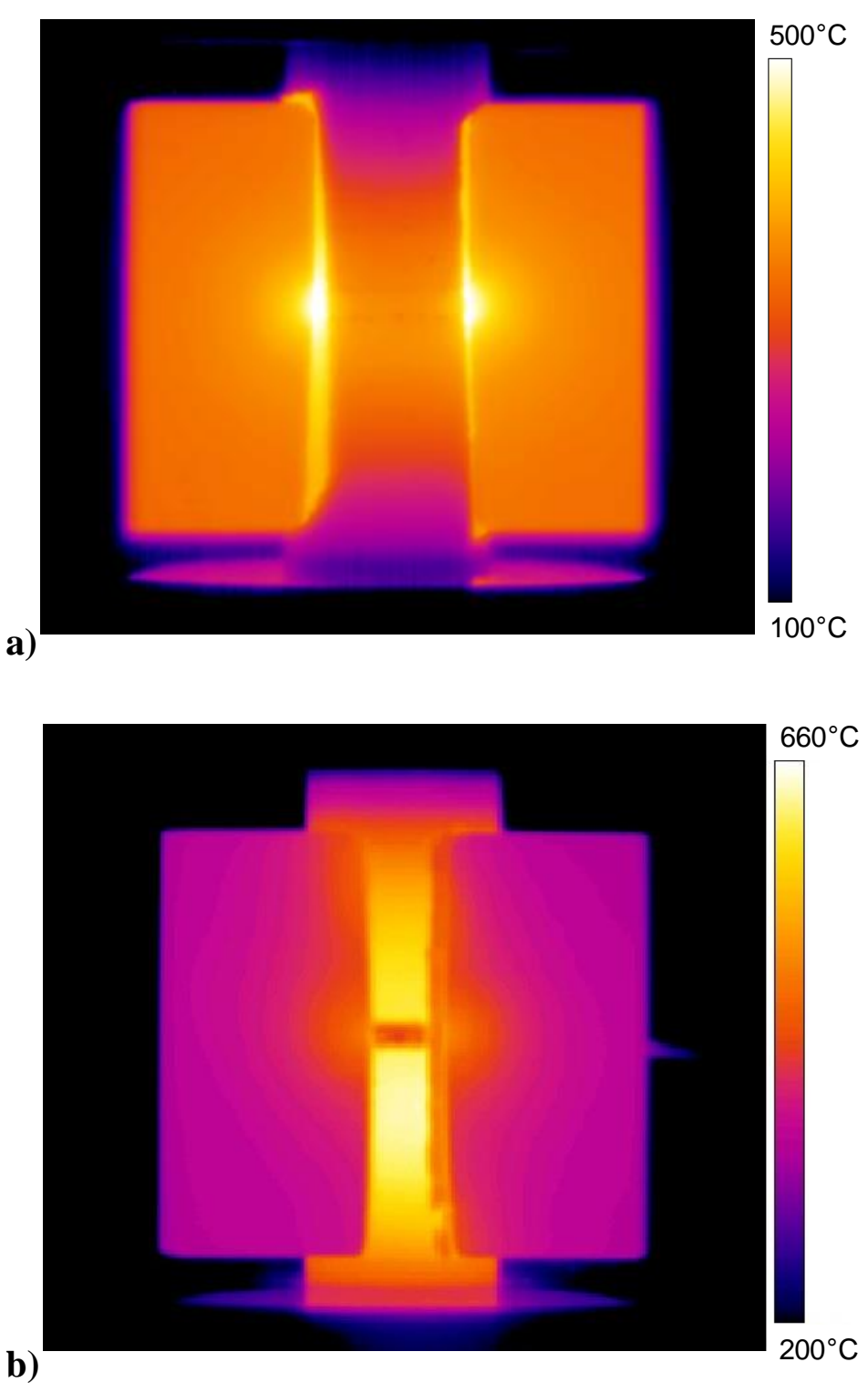
Fig.2 (2-column fitting image)

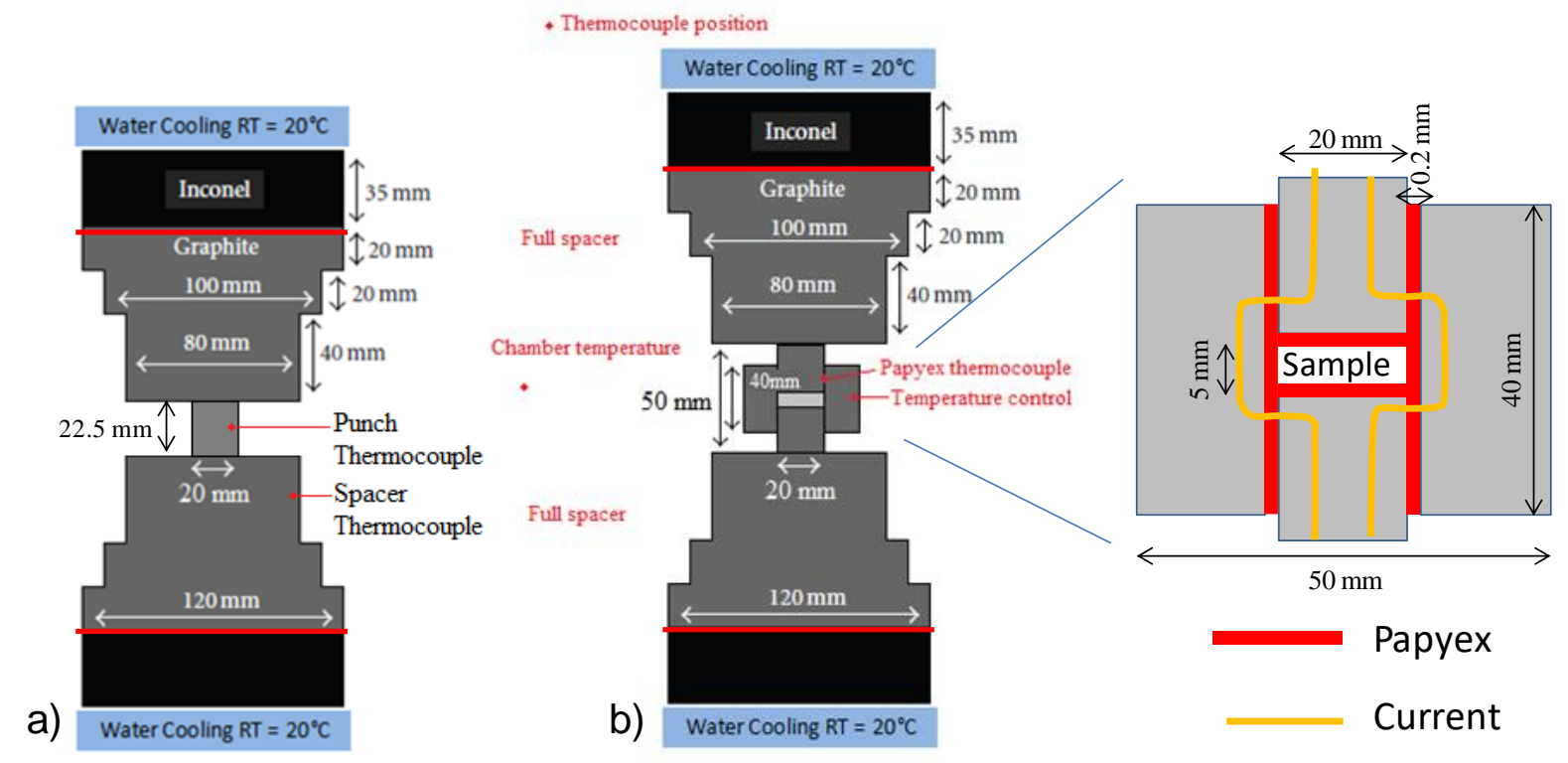


Fig.3 (2-column fitting image)

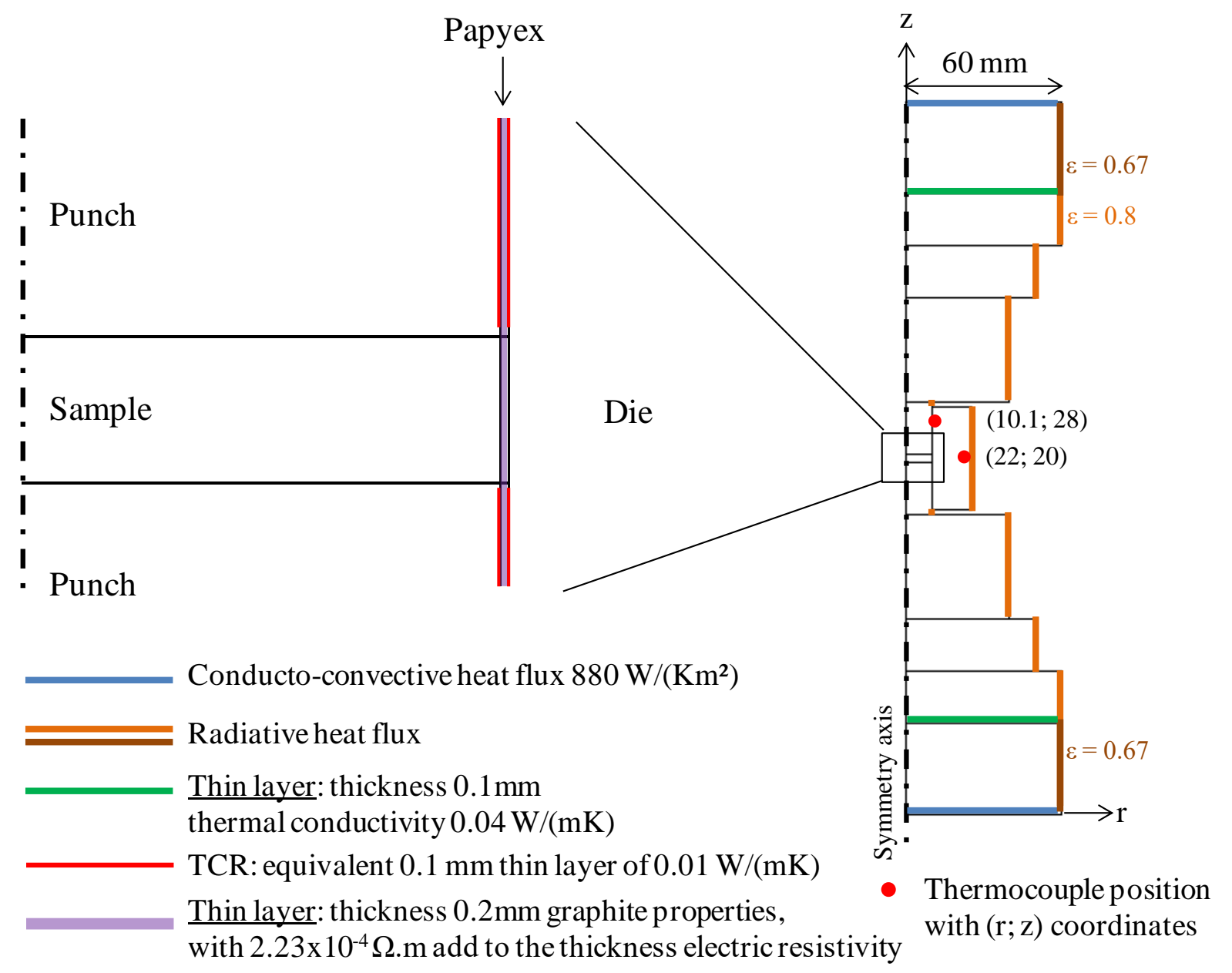


Fig. 4 (single-column fitting image)

a)

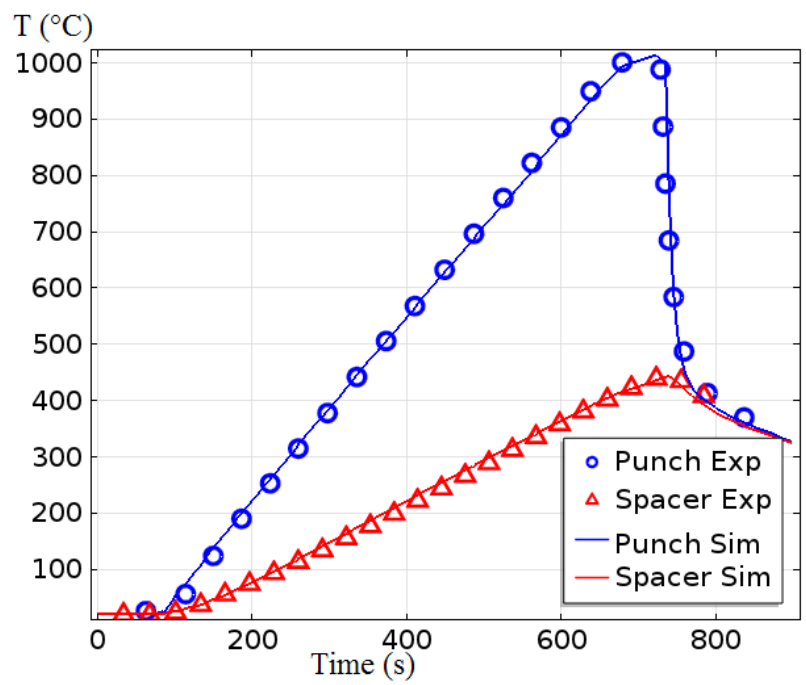

b)

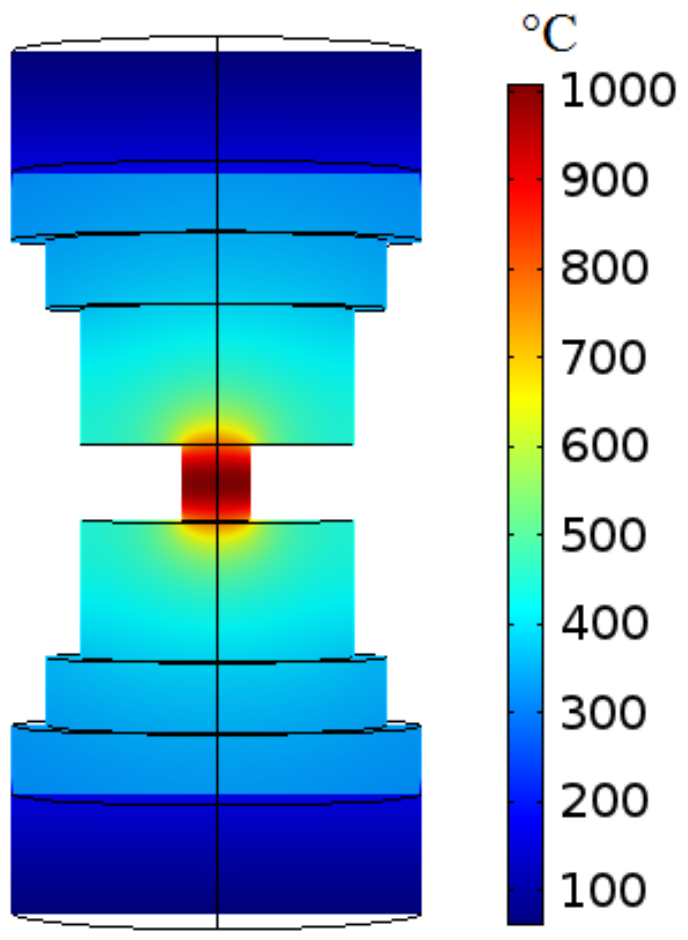


Fig. 5 (single-column fitting image)

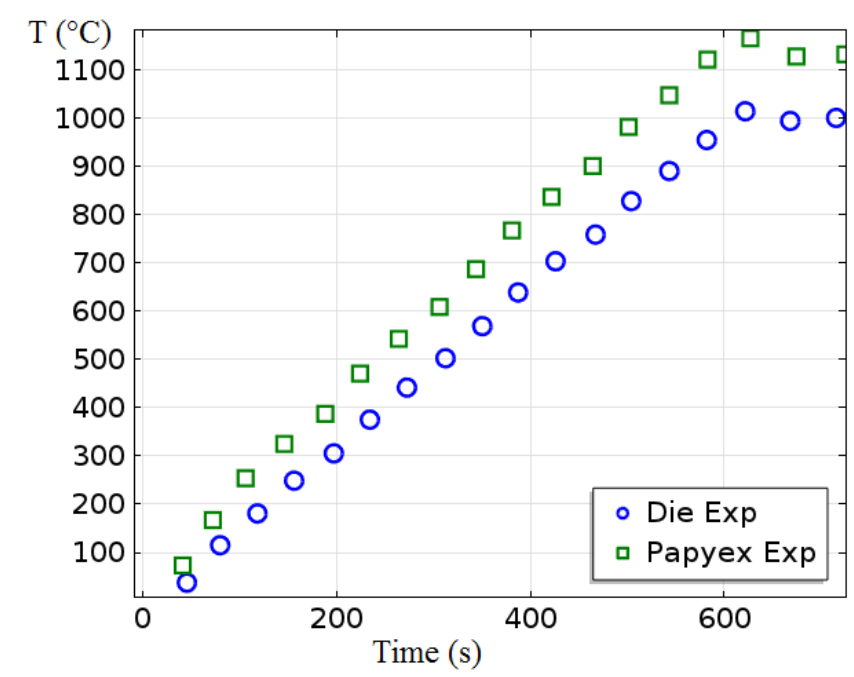


Fig.6 (2-column fitting image)

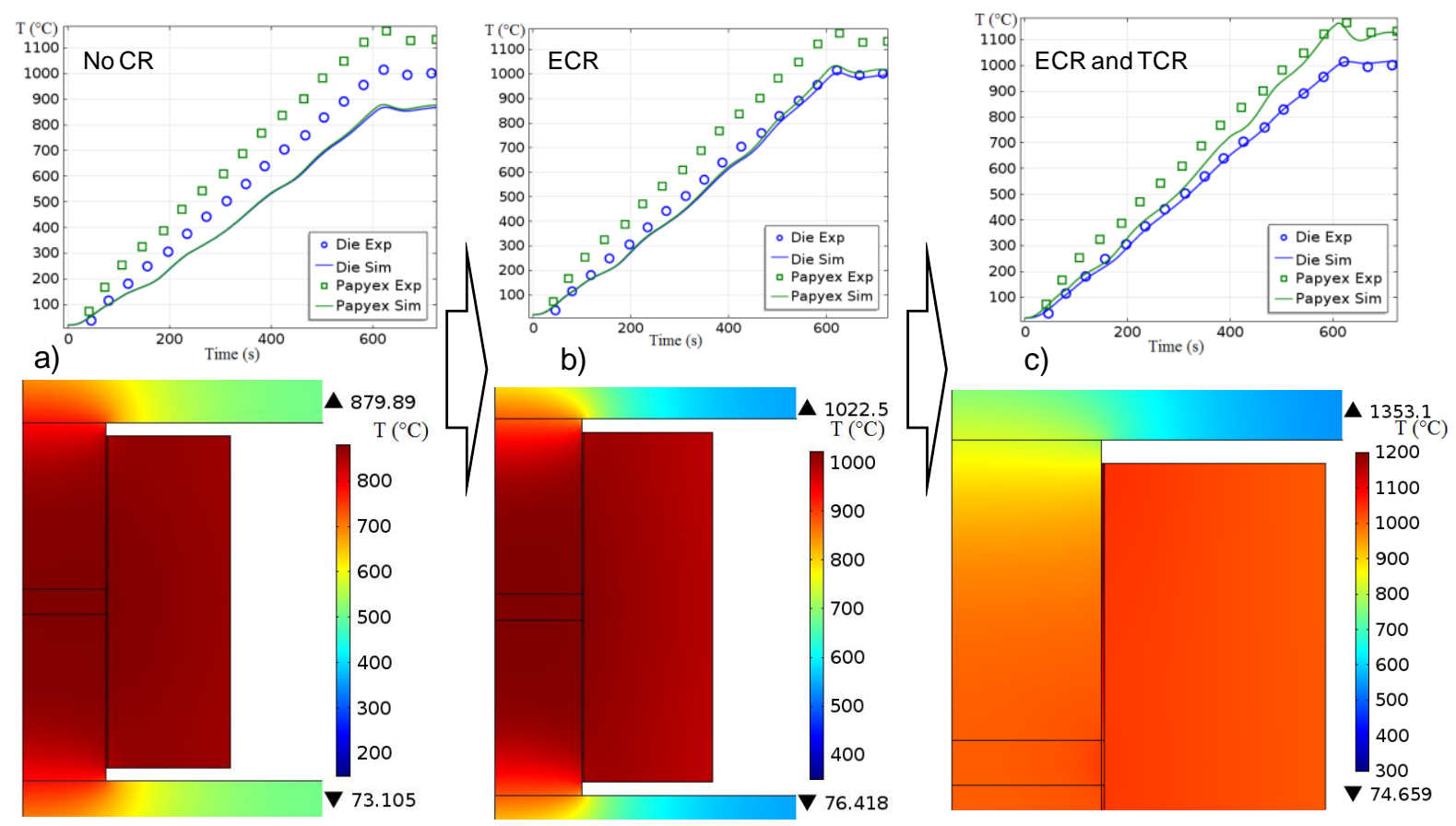


Fig.7 (2-column fitting image)

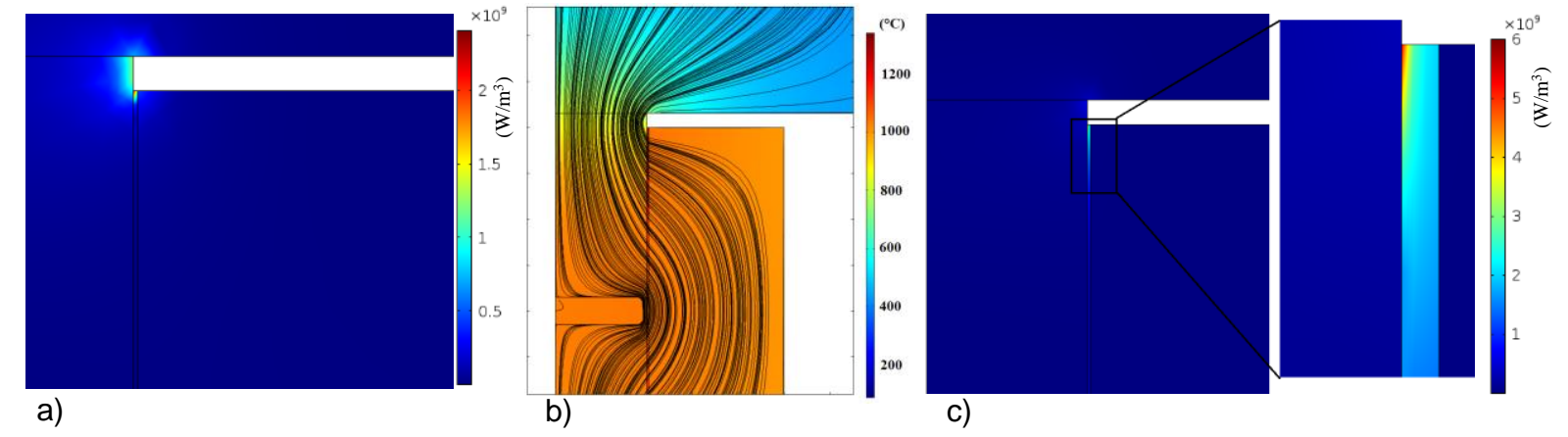


Fig. 8 (single-column fitting image)

a)

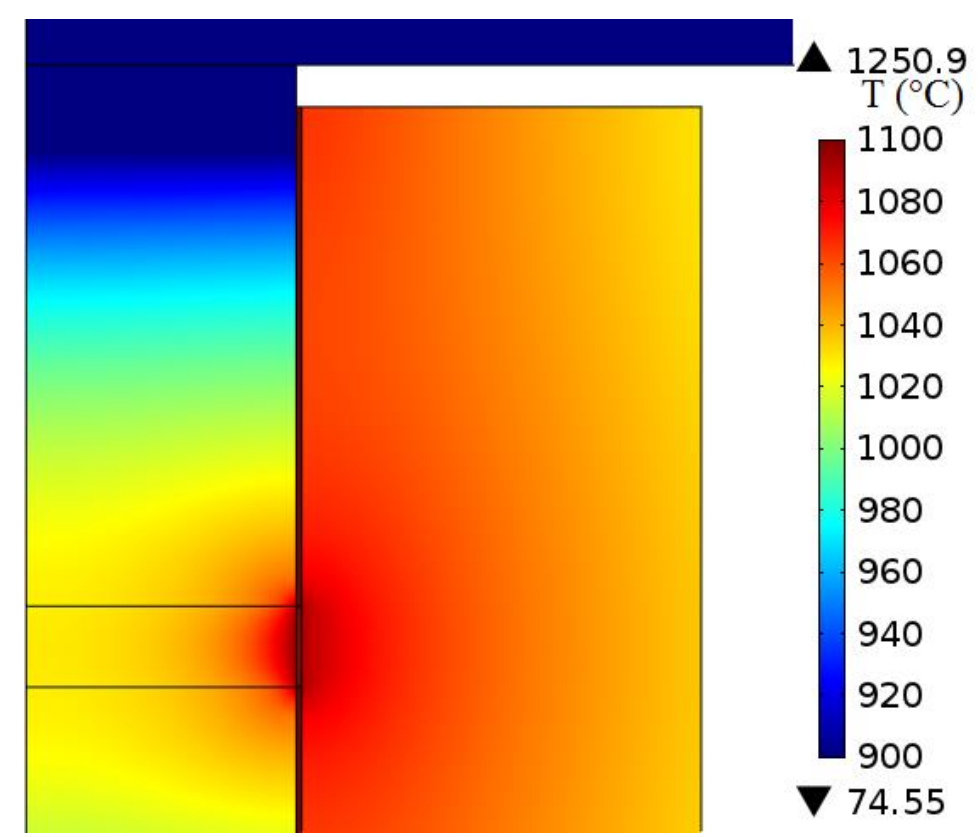

b)

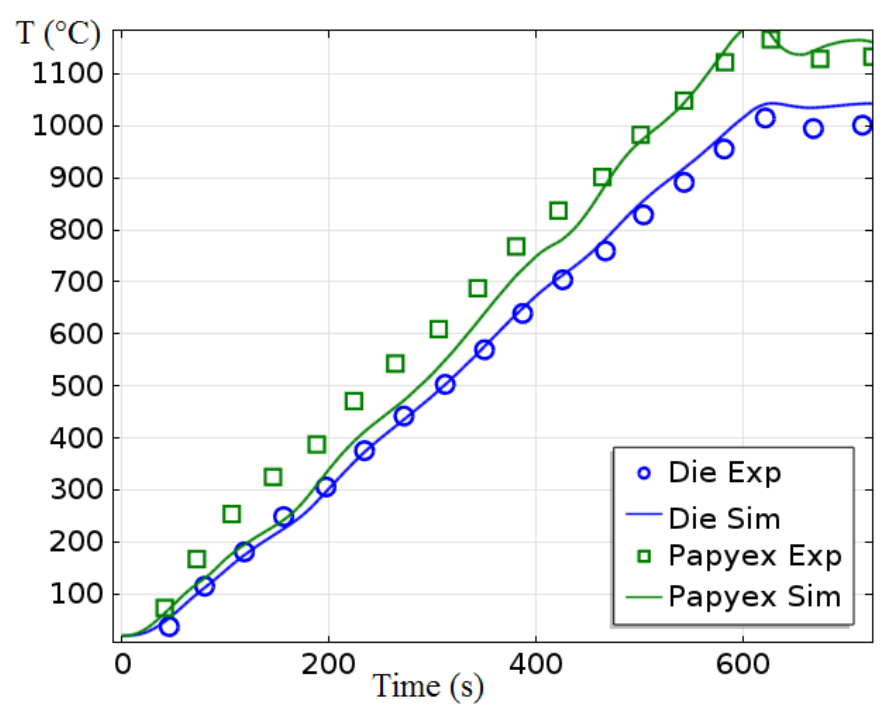


Fig. 9 (single-column fitting image)

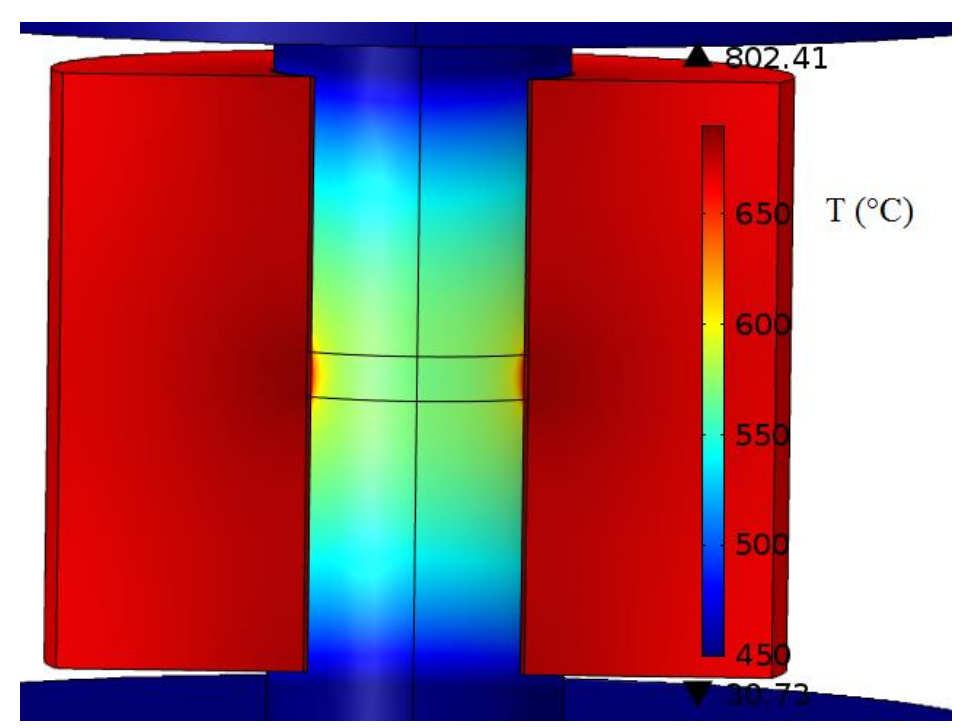

